\title{
LOOKING FOR THE ARTERY OF ADAMKIEWICZ: A QUEST TO MINIMIZE PARAPLEGIA AFTER OPERATIONS FOR ANEURYSMS OF THE DESCENDING THORACIC AND THORACOABDOMINAL AORTA
}

Randall B. Griepp, $\mathrm{MD}^{\mathrm{a}}$

M. Arisan Ergin, $\mathrm{MD}, \mathrm{PhD}^{\mathrm{a}}$

Jan D. Galla MD, $\mathrm{PhD}^{\mathrm{a}}$

Steven Lansman, $\mathrm{MD}, \mathrm{PhD}^{\mathrm{a}}$

Nguyen Khan, MD ${ }^{\mathbf{a}}$

Cid Quintana, $\mathrm{MD}^{\mathrm{a}}$

Jock McCollough, $\mathrm{MD}^{\mathrm{a}}$

Carol Bodian, $\mathrm{PhD}^{\mathrm{b}}$

\begin{abstract}
All patients undergoing resection of thoracic or thoracoabdominal aneurysms at Mount Sinai Hospital since November 1993 had spinal cord function monitored with somatosensory-evoked potentials as part of a multimodality approach to reducing spinal cord injury. In the segment to be resected, each pair of intersegmental vessels was sequentially clamped, and they were subsequently sacrificed only if no change in somatosensory evoked potentials occurred within 8 to 10 minutes after occlusion. Adjunctive protective measures included mild hypothermia $\left(31^{\circ}\right.$ to $\left.33^{\circ} \mathrm{C}\right)$, distal perfusion, corticosteroids, maintenance of high normal blood pressures, avoidance of nitroprusside, and cerebrospinal fluid drainage. Ninety-five consecutive patients operated on since 1993 (group II) were compared with 138 earlier patients (group I). Preoperative characteristics such as age, sex, etiology of aneurysm, emergency operation, and reoperation did not differ between groups, nor did operative variables such as incidence of rupture and extent of resection. Group I had slightly more smokers and slightly fewer hypertensive individuals. Group II patients had a significantly better outcome with respect to in-hospital mortality $(10.5 \%$ vs $18 \%, p=0.045)$ and paraplegia $(2 \%$ vs $8 \%, p=$ 0.008). By multivariate analysis, rupture and diabetes were associated with significantly higher in-hospital mortality, and smoking greatly increased the incidence of paraplegia. The extent of the aneurysm was a major determinant of mortality and paraplegia. The low paraplegia rate in group II was achieved without reattachment of a single intercostal or lumbar artery. No patient with fewer than 10 intersegmental arteries severed had paraplegia, and spinal cord ischemia was reversible in three patients after adjunctive maneuvers were performed to improve perfusion, suggesting that spinal cord blood supply is unlikely to depend on a single "artery of Adamkiewicz." (J Thorac Cardiovasc Surg 1996;112:1202-15)
\end{abstract}

$\Lambda$ s part of an ongoing effort to lower the incidence A of paraplegia after operations to resect aneurysms of the descending and thoracoabdominal

From the Departments of Cardiothoracic Surgery ${ }^{\mathrm{a}}$ and Biomathematics, ${ }^{\text {b }}$ Mount Sinai Medical Center, New York, N.Y.

Read at the Seventy-sixth Annual Meeting of The American Association for Thoracic Surgery, San Diego, Calif., April 28-May 1, 1996.

Received for publication May 6, 1996; revisions requested June 11, 1996; revisions received July 5, 1996; accepted for publication July 11, 1996.

Address for reprints: Randall B. Griepp, MD, Department of Cardiothoracic Surgery, Mount Sinai Medical Center, One Gustave Levy Place, New York, NY 10029.

Copyright $\mathbb{C} 1996$ by Mosby-Year Book, Inc.

$0022-5223 / 96 \$ 5.00+0 \quad \mathbf{1 2 / 6 / 7 6 3 3 4}$ aorta, we retrospectively analyzed the outcomes of all patients who have undergone operations for these lesions at Mount Sinai Hospital since 1986. During the 10 years encompassed by this study, our techniques for resection of these aneurysms have undergone a number of modifications, resulting in gradual improvement in operative mortality rate and a progressive decrease in the incidence of spinal cord injury.

The most significant change during the interval under scrutiny was the introduction in 1993 of routine intraoperative and postoperative monitoring of spinal cord function by means of somatosensoryevoked potentials (SSEPs). On the basis of our own laboratory investigations and those of others, ${ }^{1-3}$ we 
were confident that measurement of SSEPs during a short interval of temporary occlusion would allow us to determine whether a segmental vessel was critical to spinal cord function, and that subsequent sacrifice of vessels for which no change in SSEPs was observed after temporary occlusion would be tolerated. We instituted this policy of stepwise evaluation and sacrifice of segmental vessels, coupled with postoperative monitoring of SSEPs and intrathecal pressure, in November 1993. We have since used SSEP monitoring to guide intraoperative and postoperative management of spinal cord perfusion in 95 cases. This study compares the recent patients operated on under the new protocol with 138 consecutive patients operated on before 1993, for whom rigorous intraoperative and postoperative assessment of spinal cord function was not routinely carried out.

At the same time we instituted SSEP monitoring, we also modified our operative procedure in several ways to enhance spinal cord perfusion and to minimize spinal cord ischemia. These modifications also distinguish the more recent cohort of patients from those operated on earlier. First, we began to pay increased attention to the maintenance of intraoperative blood pressure at a high normal level and to avoid the use of vasodilators, instead using partial exsanguination and rapid reinfusion of blood from the reservoir of our perfusion circuit to control blood pressure. We became more strict about ensuring that mild hypothermia $\left(32^{\circ}\right.$ to $\left.33^{\circ} \mathrm{C}\right)$ prevailed during the sacrifice of intersegmental arteries and during the interval of aortic crossclamping. We began sacrificing intersegmental arteries before incising the aneurysm, thus preventing backbleeding from open intersegmental arteries and potential steal from the anterior spinal artery. With the knowledge that we would want to keep blood pressures relatively high after operation without increasing the risk of hemorrhage, we changed almost entirely to the use of full-thickness anastomosis rather than endoaneurysmal technique, and we also became extremely meticulous about ensuring hemostasis.

Since the institution of routine intraoperative SSEP monitoring and the changes in operative technique described here, we have never observed acute changes after serial temporary occlusion of segmental vessels. We therefore have not reattached intercostal or lumbar arteries in any patients in the recent group. The absence of any evidence of spinal cord ischemia after occlusion of intersegmental arteries during aneurysm resection has made us question whether there is in fact an "artery of Adam- kiewicz" whose integrity is essential for spinal cord function. We are more inclined to believe that there is a functionally continuous anterior spinal artery stretching from the foramen magnum to the cauda equina with multiple inputs throughout its length, and that no single segmental input is absolutely required for maintenance of spinal cord integrity.

Our experience supports the existence of multiple inputs into the anterior spinal artery, a situation that usually allows serial sacrifice of segmental vessels without spinal cord ischemia, either during aortic crossclamping or thereafter. The situation is precarious, however, especially if a large number of intercostal and lumbar arteries are sacrificed; ischemia can be avoided only if there is enough difference between intrathecal pressure and arterial pressure reaching the anterior spinal artery for adequate flow to occur. Spinal cord perfusion can be enhanced by increasing arterial pressure, by minimizing runoff from collateral vessels into low-pressure areas that compete for flow with the spinal cord, and by lowering intrathecal pressure.

Even when intraoperative spinal cord ischemia is unavoidable, it may not result in irreversible spinal cord injury if its duration is short and its severity is mild. Spinal cord injury is less likely to occur if the metabolic rate during ischemia is low and if reperfusion of the spinal cord after operation is sustained at adequate levels without lapses. To reduce metabolic demand, we use at least mild hypothermia during descending aortic operations in all cases and use deep hypothermia with complete circulatory arrest in some. To ensure sustained reperfusion after operation, we monitor postoperative intrathecal pressures for at least 24 hours, and cerebrospinal fluid (CSF) drainage is used to prevent CSF pressure from rising above 10 to $15 \mathrm{~mm} \mathrm{Hg}$.

Use of SSEP monitoring to assess both intraoperative and postoperative spinal cord function has been invaluable in monitoring the adequacy of spinal cord perfusion both during and after operation. It has enabled manipulation of the various factors contributing to spinal cord perfusion whenever signs of spinal cord ischemia have been noted. These new techniques have contributed to significant decreases in mortality and in the incidence of paraplegia among our more recent patients when compared with an earlier cohort who underwent operations on the descending and thoracoabdominal aorta without the benefit of spinal cord function monitoring or as scrupulous a focus on the maintenance of spinal cord perfusion. 


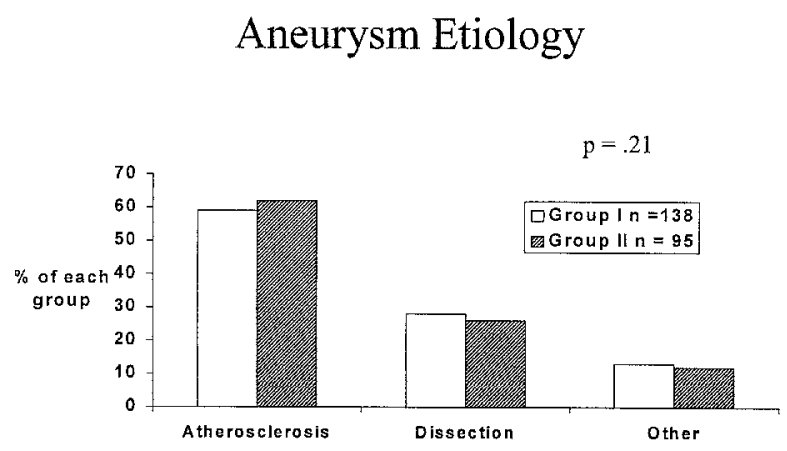

Fig. 1. Comparison of group I and group II patients with regard to etiology of aneurysms. Group I includes all patients operated on between January 1986 and October 1993. Group II includes all patients operated from November 1993, at which time SSEP monitoring and various modifications of surgical technique were initiated, until February 1996. There were 82 atherosclerotic aneurysms in group I versus 59 in group II; 38 dissections in group I versus 25 in group II; and 18 aneurysms with diverse other causes in group I versus 11 in group II. Statistical analysis showed that proportion of aneurysms of each type did not differ significantly between groups, suggesting that differences in outcome are unlikely to arise from differences in underlying lesions in each group, rather than from differences in operative strategy.

\section{Materials and methods}

Patient population. All patients undergoing resective therapy of the descending thoracic or thoracoabdominal aorta for aneurysmal disease at the Mount Sinai Medical Center between January 1986 and February 1996 were included in the study. One hundred thirty-eight patients in group I underwent aneurysm operations before November 1993, when SSEP monitoring became available at our institution, and 95 patients in group II underwent operation with SSEP monitoring after that time.

The groups were not significantly different with respect to a number of variables previously shown to have an impact on outcome in patients with aneurysmal disease, as shown in Table I. Specifically, the earlier and more recent groups of patients did not differ significantly with respect to age, sex, proportion of emergencies, ruptures or reoperations, or percentage of patients with diabetes. The proportion of patients with a significant history of hypertension was higher in the more recent group, and a history of smoking was more frequent in the earlier group.

The aneurysms also did not differ significantly in etiology between the groups, as seen in Fig. 1. Aneurysms were atherosclerotic in origin in almost $60 \%$ of each group; slightly more than one quarter in both groups had dissections, and one eighth had lesions with diverse other etiologies.

The extent of the lesions did not differ statistically between the two groups, as shown in Fig. 2, although there was a trend toward higher risk and more extensive lesions
Table I. Patient characteristics

\begin{tabular}{lccc}
\hline & $\begin{array}{c}\text { Group I } \\
(n=138)\end{array}$ & $\begin{array}{c}\text { Group II } \\
(n=95)\end{array}$ & $p$ \\
\hline Age (yr) & 63 & 65 & 0.40 \\
Male (\%) & 64 & 57 & 0.24 \\
Hypertension (\%) & 45 & 61 & 0.02 \\
History of smoking (\%) & 62 & 49 & 0.05 \\
Diabetes (\%) & 4 & 5 & 0.75 \\
CAD (\%) & 20 & 28 & 0.15 \\
Reoperation (\%) & 27 & 24 & 0.66 \\
Emergency operation (\%) & 22 & 18 & 0.47 \\
Rupture (\%) & 29 & 22 & 0.24 \\
\hline
\end{tabular}

$C A D$, Coronary artery disease.

in the more recent group of patients. The distribution of lesions, arranged according to the number of intersegmental arteries sacrificed, is shown for each patient group in Fig. 3. From $56 \%$ to $57 \%$ of patients in both groups I and II had lesions involving segments below T9, indicating that a majority in each group had lesions posing a relatively high risk for paraplegia.

The two groups did differ significantly with respect to operative technique and perfusion methods (Fig. 4). More of the later group were operated on with profound hypothermia and circulatory arrest (38\% vs $25 \%$ ), and fewer of the later group had no distal perfusion. The higher percentage of the later group who underwent hypothermic circulatory arrest (HCA) probably reflects an increasing reluctance to place clamps anywhere near the arch vessels because of the danger of cerebral embolization. Because monitoring of SSEPs requires perfusion, the proportion of patients who had no form of distal perfusion dropped from $14 \%$ in the earlier group to $3 \%$ in the more recent cohort. The number who had some form of distal perfusion except during the performance of an open distal anastomosis remained the same. The differences in operative technique probably explain differences in perfusion parameters; there were no significant differences in mean crossclamp times between the earlier and later groups (53.8 vs 53.4 minutes, $p=0.92$ ). The earlier group also differed from the more recent group in having slightly but significantly higher minimum esophageal and rectal temperatures during aortic crossclamping than the later group (Fig. 5).

SSEPs. SSEP monitoring is performed with a Cadwell Quantum 84 generator/stimulator (Cadwell Laboratories, Kennewick, Wash.). Stimuli are applied at both ankles overlying the posterior tibial nerves, and potentials are recorded at multiple loci on the scalp by means of skin needle electrodes. Average signals of 200 potentials, cycled alternately between the left and right lower extremities, are generated and displayed both as wave forms and as digital expressions of latency measurements. Continuous monitoring is initiated immediately after induction of anesthesia and is continued throughout the operative procedure.

After operation, monitoring is carried out hourly until the patient is awake and can move both lower extremities on command. On several occasions, SSEP monitoring has been reinstituted later in the postoperative course in response to clinical evidence of spinal cord ischemia. 
Aneurysm Extent (conventional classification)

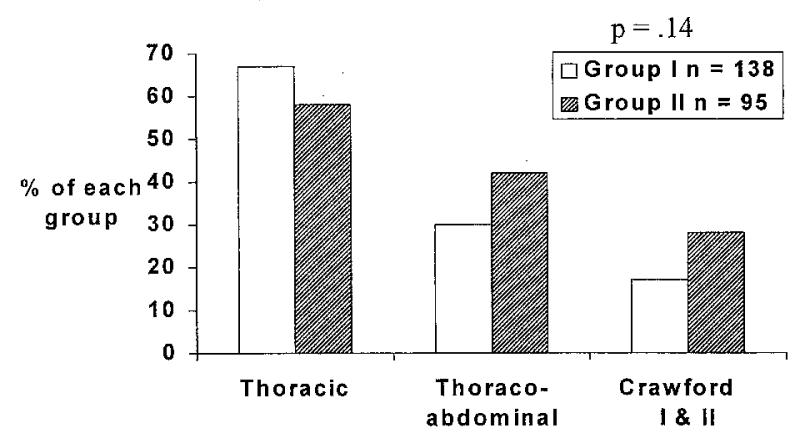

Fig. 2. Comparison between group I and group II patients (defined in Fig. 1 and in text) with respect to extent of aneurysms, categorized according to conventional classification schemes for assessing risk of spinal cord injury after operation. Thoracic aneurysms were defined as those aneurysms that end above the diaphragm and are therefore associated with low risk of spinal cord injury; 92 patients in group I and 55 in group II had such aneurysms. Thoracoabdominal aneurysms include all lesions extending from the descending aorta into the abdomen and are most likely to result in spinal cord injury: 46 patients in group I and 40 in group II had aneurysms thus classified. Crawford $I$ and $I I$ aneurysms are those that fit into groups defined by Stanley Crawford; these are the subset of thoracoabdominal aneurysms in which paraplegia is most likely to develop after operation. There were 18 Crawford I and six Crawford II aneurysms in Group I, for a total of 24; there were 20 Crawford I and seven Crawford II aneurysms in group II, for a total of 27 . The $p$ value indicates that proportions of patients falling into these conventional categories of aneurysm extent-which define risk of spinal cord injury-did not differ significantly between groups I and II, making it valid to compare incidence of paraparesis and paraplegia after operation.

SSEP latencies usually increase $5 \%$ to $10 \%$ as the temperature drops from $37^{\circ}$ to $33^{\circ} \mathrm{C}$ during the initial portion of the procedure. Signals from the left leg are routinely lost after cannulation of the left femoral artery; signals from both legs usually disappear within 5 minutes of discontinutation of distal bypass during performance of open distal anastomoses. With restoration of distal perfusion and relief of peripheral nerve ischemia, SSEP signals return.

\section{Operative technique}

Incision and exposure. Patients are placed in the left lateral decubitus position, with the hips swiveled posteriorly to facilitate exposure and cannulation of the femoral artery and if necessary the femoral vein. Lesions in the upper chest are exposed through the fourth or fifth intercostal space, and those in the lower chest or abdomen are exposed through the sixth or seventh interspace. One

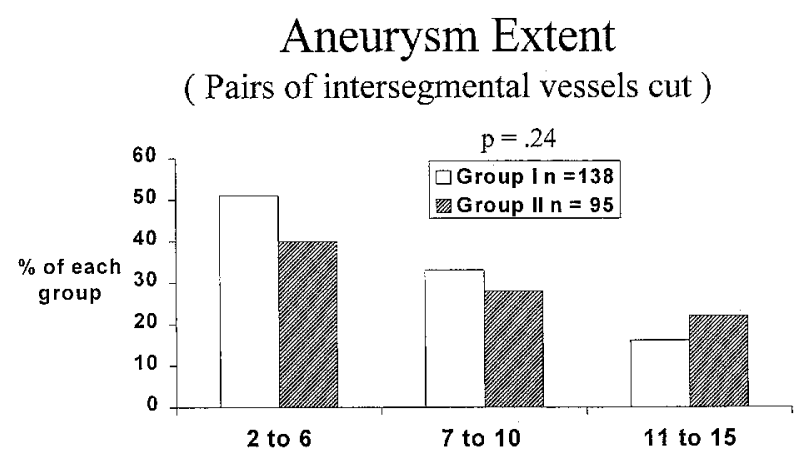

Fig. 3. Comparison between group I and group II patients (defined in Fig. 1 and in text) with respect to extent of aneurysms defined by number of intersegmental (intercostal and lumbar) vessels severed during aneurysm operation, which we propose as a more accurate way of defining risk of postoperative spinal cord injury. Seventy patients in group I and 36 patients in group II had fewer than seven arteries sacrificed; 46 patients in group I and 36 in group II had fewer than 11 vessels cut, and 22 patients in group I and 21 in group II had 11 or more arteries cut, putting them into the group at highest risk. The proportion of patients with more extensive aneurysms appears slightly greater in group II, but this difference was not statistically significant, as indicated by the $p$ value.

or two ribs above and below the intercostal incision are transected as needed to improve exposure.

In thoracoabdominal incisions, the seventh interspace entry is extended across the costal margin to the lateral border of the rectus abdominis muscle. Care is taken to preserve the integrity of the left internal thoracic-epigastric arterial axis to maintain anterior perfusion of the intersegmental arteries. When exposure of the entire abdominal aorta is required, the diaphragm is incised circumferentially and dissection is carried out in the retroperitoneal plane posterior to the aorta. If exposure of only the upper abdominal aorta is required, a short radial incision of the diaphragm beginning at the aortic hiatus is used.

Mobilization of aorta and sacrifice of intercostal and lumbar arteries. Beginning at the site of the anticipated proximal anastomosis, the aorta is mobilized circumferentially. Intercostals are exposed and temporarily occluded with a large Ligaclip Extra (Ethicon, Inc., Somerville, N.J.); usually one or two pairs are occluded simultaneously. If no changes in the latency of the SSEPs are observed during an interval of 8 to 10 minutes, additional clips are applied and the segmental vessels are transected. The entire aneurysm is mobilized in this way from its proximal to its distal margins, with sequential clamping and then sacrifice of the intersegmental arteries during a cumulative interval of 30 to 90 minutes.

Perfusion and anastomoses. Distal perfusion is carried out with a Bio-Medicus pump (Bio-Medicus, Inc., Eden Prairie, Minn.), with a reservoir to allow rapid blood withdrawal and reinfusion. Low-dose heparinization, 100 $\mathrm{U} / \mathrm{kg}$, is used. The proximal cannulation site is the left 


\section{Types of Perfusion}

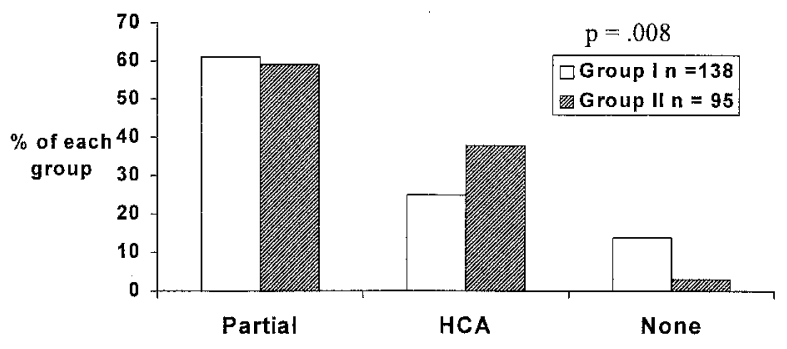

Fig. 4. Comparison between group I and group II patients (defined in Fig. 1 and in text) with respect to use of distal perfusion during repair of thoracic and thoracoabdominal aneurysms. Eighty-four patients in group I versus 56 in group II had partial bypass, 35 in group I versus 36 in group II had HCA, and 19 in group I versus three in group II had no distal bypass. Although the proportion of patients who had partial perfusion-distal perfusion without HCA - was the same in both groups, there were significant differences in proportions of group I and group II patients who underwent HCA and in whom no distal perfusion was used. This results in a highly significant difference between groups, as indicated by the $p$ value, with respect to distal perfusion methods, which reflects acknowledged differences in surgical techniques between groups.

ventricular apex, left atrial appendage, or the left inferior pulmonary vein, with the last being preferred. In most cases, distal perfusion is through the left femoral artery, but the distal aorta can be used in cases of severe femoral artery disease. The perfusion rate ranges from 1500 to $3500 \mathrm{ml} / \mathrm{min}$ and is adjusted to equalize proximal and distal mean blood pressures.

In patients in whom an open distal anastomosis is not utilized, clamps are applied to the aorta proximal and distal to the aneurysm, and the aneurysm is removed in toto. A protein-impregnated Dacron polyester fabric graft (Hemashield, Meadox Medicals, Inc., Oakland, N.J.) of appropriate size is anastomosed to the aorta with a continuous 3-0 Prolene (Ethicon, Inc., Somerville, N.J.) suture and a cuff of Teflon (polytetrafluoroethylene) felt outside the aorta, with careful positioning of the graft inside the aorta. Before completion of the second anastomosis, appropriate flushing and deairing of the proximal and distal aorta are carried out; the suture line is then completed, distal perfusion is discontinued, and the clamps are removed.

Open distal anastomoses. If a simple open anastomosis (most commonly a beveled anastomosis at the celiac trunk) is to be performed, distal perfusion is interrupted and the distal aortic clamp is removed. Adjustment of proximal blood pressure to a high normal level (mean 80 to $100 \mathrm{~mm} \mathrm{Hg}$ ) is accomplished by opening the proximal aortic clamp slightly, resulting in partial exsanguination. The distal anastomosis is then carried out as described previously.

\section{Minimum Temperature (HCA patients excluded)}

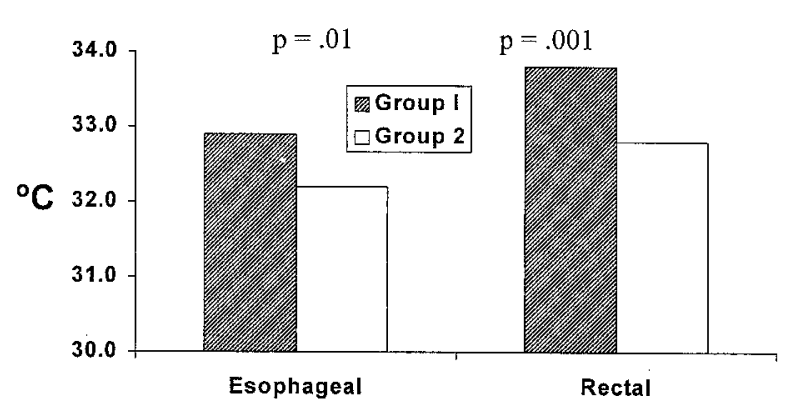

Fig. 5. Comparison between group I and group II patients (defined in Fig. 1 and in text) with respect to minimum temperatures recorded during sacrifice of intersegmental arteries and during aortic crossclamping. $\mathrm{Pa}-$ tients who had HCA are excluded from both groups. As indicated by $p$ values, both esophageal and rectal temperatures were slightly but significantly lower in group II patients, in whom a greater emphasis was placed on minimizing spinal cord metabolism during operation.

If the entire abdominal aorta is to be replaced but there is still enough normal distal aorta to allow clamping at the bifurcation, a clamp is placed at this level and distal perfusion is continued to provide spinal cord perfusion through the hypogastric arteries. Carrel patches of the abdominal branches, either singly or in combinations dictated by proximity, are then prepared. Extensive backbleeding, particularly from the celiac axis and the superior mesenteric arteries, is prevented with balloon-tipped catheters. The celiac, superior mesenteric, and right renal arteries are then attached to one or more openings in the side of the aortic graft by means of 3-0 Prolene sutures and Teflon felt buttressing. The clamp is then moved distally on the graft, restoring perfusion to these visceral vessels while the anastomosis of the graft to the abdominal aorta is carried out. On completion of the distal anastomosis, removal of the clamp restores antegrade lower extremity perfusion. The left renal artery is then attached to the graft with a rubber-shod, side-biting clamp.

Use of HCA. HCA is used in several different situations: when the entire aortic arch, or a portion of it, is to be resected in continuity with a descending thoracic aortic aneurysm; when the aneurysm arises so close to the left subclavian artery that a clamp cannot be applied to the aorta without threatening the flow into the subclavian artery, which makes an important contribution to spinal cord blood supply; and when the aorta is filled with potentially embolic atheromatous material that might be dislodged retrogradely into the cerebral circulation. Under these circumstances, when use of HCA is anticipated, mobilization of the aneurysm and serial sacrifice of the intersegmental vessels with SSEP monitoring are carried out before cooling as described previously. 
When the entire segment to be resected has been mobilized and its intersegmental arteries have been serially severed, full-dose heparinization $(300 \mathrm{U} / \mathrm{kg})$ is initiated, cannulation for cardiopulmonary bypass is performed, and central cooling is begun. Venous cannulation is effected with a long catheter placed through the left femoral vein into the right atrium with the help of a guide wire and transesophageal ultrasonography. Arterial cannulation is carried out through the left femoral artery. The left side of the heart is decompressed after the occurrence of ventricular fibrillation through the left inferior pulmonary vein or the left ventricular apex. When adequate cooling (as determined by the length of anticipated arrest) has been achieved, the patient's head is packed in ice, perfusion is discontinued, and the aneurysm is opened at the level of the anticipated proximal anastomosis.

An appropriate cuff of aorta is prepared and the proximal anastomosis is carried out. Air is then fiushed from the aortic arch and the heart by flowing from the cardiopulmonary bypass circuit into the right atrial catheter, and an arterial perfusion catheter is placed in the graft just distal to the anastomosis. When deairing is complete, the graft is clamped distally and cold perfusion of the proximal circulation is reinstituted.

The distal anastomosis or anastomoses can be carried out with the open technique with no perfusion of the hypothermic lower body, or a clamp can be applied beyond the distal extent of the aneurysm and perfusion of the lower body can then be maintained through the femoral artery catheter. Warming is begun whenever the entire body can be perfused.

Postoperative monitoring of spinal cord function. All patients have monitoring of SSEPs for at least 12 hours after operation as previously described in detail, longer for patients who awaken more slowly. Each patient also has a spinal catheter placed after operation for hourly monitoring of intrathecal pressure and for drainage of CSF if required. CSF is drained if the intrathecal pressure rises above $10 \mathrm{~mm} \mathrm{Hg}$, even in the absence of any signs of spinal cord dysfunction. Once the patient is awake, spinal cord motor and sensory function are assessed clinically on an hourly basis. If any change is noted, spinal cord perfusion pressure is optimized by draining CSF to lower intrathecal pressure and by increasing arterial pressure, and SSEP monitoring is often reinstituted.

Statistical methods. Risk factors for all patients in both groups I and II were screened for possible association with in-hospital mortality, including deaths in the operating room, by univariate analysis with $\chi^{2}$ tests or $t$ tests, as appropriate. The same factors were then screened in the same way for association with paraplegia. Tests $\left(\chi^{2}\right.$ and $t$ tests) were also done to identify important changes in the occurrence rate of any factors after October 1993, the date after which patients were classified in group II as having had recent operations. Variables with significance levels of 0.25 of less were retained for further analysis by multivariate logistic regression.

The first variables entered in the logistic regression analysis were factors known before the operation was begun. Operative variables were entered next. Finally, a variable for recent operations was introduced. Consideration of any influence of esophageal and rectal tempera-

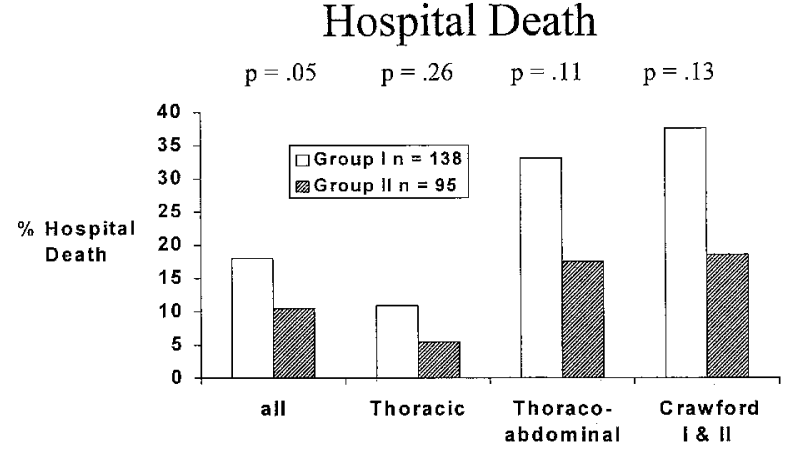

Fig. 6. Comparison between group I and group II patients (defined in Fig. 1 and in text) with respect to in-hospital mortality after aneurysm resection. All includes all patients in each group; other categories are conventional classifications reflecting aneurysm extent, as defined in Fig. 2. There is a significantly lower mortality after operation among patients undergoing operation more recently (group II) when all patients are included: 25 patients in group I died, versus 10 in group II. When smaller numbers of patients in other categories are compared, however, differences are not significant; 10 patients with thoracic aneurysms died in group I versus three in group II; 15 with thoracoabdominal aneurysms in group I died versus seven in group II; and nine with Crawford I and II aneurysms died in group I versus five in group II.

tures and of pump and crossclamp times was limited to patients who had undergone what we termed "partial perfusion," excluding patients with no distal perfusion or with HCA. Multivariate logistic analysis was carried out separately for factors associated with in-hospital mortality and for factors associated with paraplegia. Mantel-Haenszel tests were used to test for associations between specific factors and each of the outcomes (death and paraplegia), stratifying on the time of operation (recent or earlier group).

\section{Results}

Mortality. Overall mortality was $18 \%$ in the earlier group, versus $10.5 \%$ among the more recent patients $(p=0.045)$. When stratified according to extent of the aneurysm, there was a clear-cut decrease in mortality in all subgroups (Fig. 6), but none of these comparisons reached statistical significance.

When the number of segmental arteries involved was used as a measure of the extent of aneurysms (Fig. 7), there was a decrease in mortality in the later group for all aneurysms in which more than six intersegmental arteries were involved. For the subgroup with involvement of seven to 10 segmental arteries, this improvement in operative survival was statistically significant. Although patients with in- 


\section{Hospital Death}

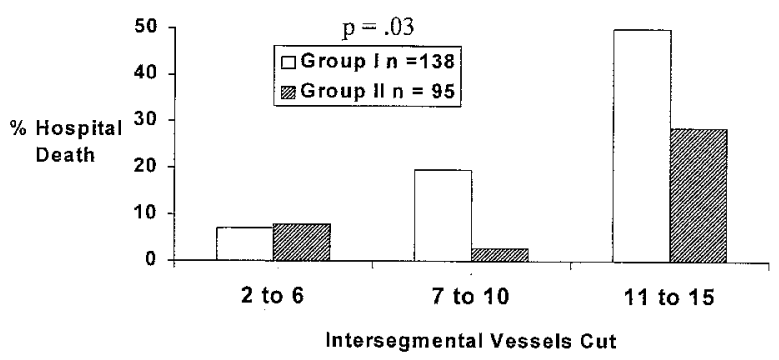

Fig. 7. Comparison between group I and group II patients (defined in Fig. 1 and in text) with respect to in-hospital mortality after aneurysm resection. When classified by extent of aneurysm as defined by number of intersegmental vessels severed, the more recent group II patients had a significantly lower mortality, not only overall ( $p=0.03$ ) but also in operations involving seven to 10 severed intersegmental arteries; nine patients in group I died versus one in group II $(p=0.02)$. Eleven patients in group I with more than 10 intersegmental vessels severed died, versus six patients in group II, and in the group with the smallest number of intersegmental vessels cut, there were five deaths in group I and three in Group II.

volvement of seven to 10 segmental arteries had a $3 \%$ mortality in the more recent cohort, the subgroup of patients with extremely extensive aneurysms - involving more than 10 segmental vesselsremained very high at $28.6 \%$.

When a multivariate analysis of all patients was undertaken to ascertain risk factors associated with higher mortality, the preoperative presence of rupture and that of diabetes both emerged as significant preoperative factors, with odds ratios as shown in Table II. Rupture was defined as blood in the pleural space or the presence of a contained hematoma. A history of smoking significantly lowered the risk of death after operation, for reasons that are not readily apparent. Other factors, such as age, sex, previous operation, the presence of hypertension or coronary artery disease, the etiology of the aneurysm, and whether the operation was performed on an emergency basis had no significant impact on mortality in multivariate analysis. The significance of emergency operation as a risk factor disappeared when the stronger predictor, rupture, was introduced.

Among operative factors, as expected, multivariate logistic regression analysis showed that patients in the more recent cohort were much less likely to
Table II. Determinants of in-hospital mortality, multivariate analysis of all patients

\begin{tabular}{lcc}
\hline Determinant & Odds ratio & $p$ \\
\hline $\begin{array}{l}\text { Pairs of intersegmental } \\
\text { arteries cut (continuous }\end{array}$ & 1.3 & 0.0001 \\
$\quad$ variable) & & \\
Pairs of intersegmental & 8.6 & 0.0001 \\
$\quad$ arteries cut ( $>9$ vs $<9)$ & & \\
History of smoking & 0.35 & 0.01 \\
New technique (group II) & 0.38 & 0.03 \\
Rupture & 2.4 & 0.04 \\
Diabetes & 4.6 & 0.06 \\
\hline
\end{tabular}

Variables not significant: HCA, minimum temperature, etiology, thoracoabdominal aneurysm, Crawford I and II classification, emergency operation, hypertension, and all other factors not significant in univariate analysis.

die after operation (Table II) than were patients operated on earlier. In addition, the mortality rate increased progressively with each pair of intersegmental arteries sacrificed. When the number of intersegmental arteries sacrificed was entered not as a continuous variable but categorically, with all patients who had more than 10 intersegmental arteries severed considered as a group, members of this group with more extensive aneurysms were 8.6 times as likely to die after operation than were those individuals with aneurysms involving fewer pairs of intersegmental arteries $(p=0.001)$. Other operative factors that differed significantly between the earlier and more recent groups of patients in univariate analysis, including use of HCA and minimum esophageal and rectal temperatures, were not independent predictors of mortality in multivariate analysis.

Paraplegia. The overall rate of paraplegia, like the mortality rate, was significantly lower in the more recent cohort of patients ( $2 \%$ in group II vs $8 \%$ in group I, $p=0.008 ;$ Fig. 8 ). This decline in the incidence of spinal cord injury was statistically significant in the subgroup of patients with Crawford I and II aneurysms $(p=0.02)$ and in the larger subgroup of patients at risk, which includes all of those with thoracoabdominal aneurysms $(p=0.04)$. In fact, among the 95 recent patients whose operations were carried out with intraoperative and postoperative spinal cord function monitoring, only those whose aneurysms spanned more than 10 intersegmental arteries had paraplegia, at a rate of 9.5\% (Fig. 9).

Multivariate analysis confirmed the fact that patients operated on since the institution of spinal cord function monitoring were much less likely to 


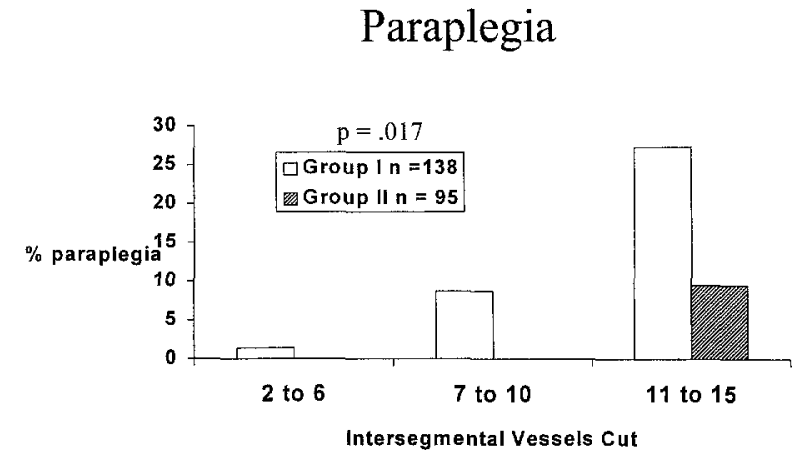

Fig. 8. Comparison between group I and group II patients (defined in Fig. 1 and in text) with respect to paraplegia after resection of descending thoracic and thoracoabdominal aneurysms. Rate of spinal cord injury is significantly lower among group II patients overall (11 patients in group I versus two in Group II), and also if thoracoabdominal aneurysms or Crawford I and II aneurysms are considered separately. Two patients with thoracic aneurysms in group $\mathrm{I}$ had spinal cord injury, versus none in group II; nine with thoracoabdominal aneurysms had paraplegia in group I, whereas only two in group II had paraplegia. In group I, five patients with Crawford I and three with Crawford II aneurysms had paraplegia; in Group II, only two patients in the Crawford I and II group, both with Crawford II aneurysms, had spinal cord injury.

have paraplegia than were those in the earlier cohort (Table III). Also as expected, the number of intersegmental arteries sacrificed was directly related to the incidence of paraplegia, with the odds ratio reflecting the fact that paraplegia is progressively more likely to occur as more intersegmental arteries are sacrificed. When a multivariate analysis is carried out to examine the impact of sacrifice of more than 10 intersegmental vessels, the odds ratio indicates that the risk of paraplegia associated with these more extensive aneurysms is 29 times the rate of paraplegia associated with cases in which 10 or fewer intercostals are involved.

Multivariate logistic regression analysis also revealed a powerful effect of smoking as a risk factor for development of paraplegia, with an odds ratio of $20.5(p=0.01)$. Multivariate analysis failed to implicate any other risk factors as important influences on the rate of paraplegia, including age, sex, etiology of the aneurysm, the presence of diabetes, hypertension or coronary artery disease, previous operations, emergency operation, rupture, use of HCA, and minimal esophageal or rectal temperature. It is interesting to note that the influence on

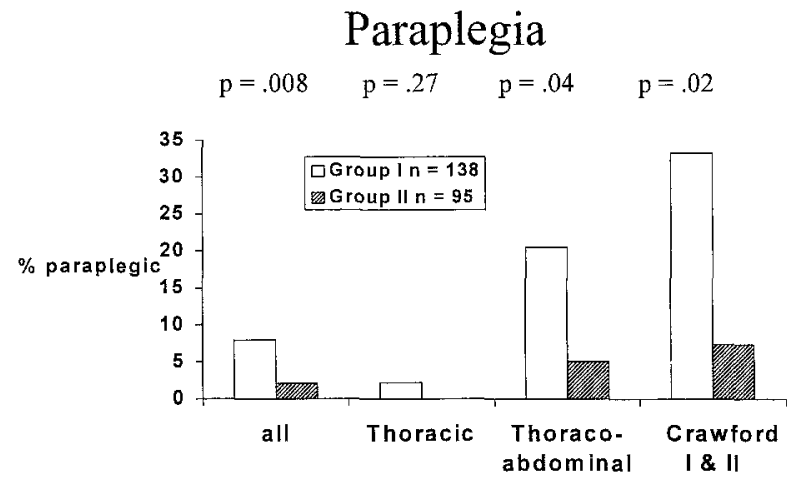

Fig. 9. Comparison between group I and group patients (defined in Fig. 1 and in text) with respect to paraplegia after resection of descending thoracic and thoracoabdominal aneurysms. When extent of aneurysm is defined by number of intersegmental vessels severed, group II patients show significantly lower rates of paraplegia in each category: only two patients in group II with more than 10 vessels severed had spinal cord injury, whereas in group I paraplegia developed in six of the patients with most extensive aneurysms, an additional four in the intermediate group, and one in the group with very few intersegmental arteries cut.

incidence of paraplegia of both the combined Crawford I and II classification, and of the group including all thoracoabdominal aneurysms, compared with lesions limited to the descending thoracic aorta, is superseded by the impact of the extent of aneurysms as defined by the numbers of pairs of intersegmental arteries sacrificed.

Irreversible paraplegia occurred in only two patients in the recent series; in each of these, 14 pairs of intersegmental arteries had been sacrificed. In one of these, complete paralysis developed immediately after operation, despite the absence of changes in SSEPs during the division of the intersegmental arteries. In the second case, paraplegia developed on the second postoperative day, after several episodes of hypotension caused by poorly controlled arrhythmias; the patient continued hemodynamically unstable, and paraplegia persisted until death several hours later.

Transient spinal cord injury was seen after operation in three patients, each of whom had between eight and 14 pairs of intersegmental arteries sacrificed. In each case, on discovery of the abnormal SSEPs or clinical evidence of spinal cord ischemia, measures were immediately instituted to improve spinal cord perfusion. These included elevating the arterial pressure and draining CSF to decrease 
Table III. Determinants of paraplegia, multivariate analysis of all patients

\begin{tabular}{ccc}
\hline \multicolumn{1}{c}{ Determinant } & Odds ratio & $p$ \\
\hline $\begin{array}{l}\text { Pairs of intersegmental } \\
\text { arteries cut (continuous } \\
\text { variable) }\end{array}$ & 1.8 & 0.0001 \\
$\begin{array}{l}\text { Pairs of intersegmental } \\
\quad \text { arteries cut (<9 vs }>9)\end{array}$ & 29 & 0.002 \\
$\quad$ History of smoking & 21 & 0.01 \\
New technique (group II) & 0.13 & 0.02 \\
\hline
\end{tabular}

Variables not significant: HCA, minimum temperature, etiology, thoracoabdominal aneurysm, Crawford I and II classification, emergency operation, rupture, hypertension, diabetes, and all other factors not significant in univariate analysis.

intrathecal pressure. Heparin was administered in two cases; steroids were also given in all three. These measures were immediately successful in reversing the evidence of spinal cord injury in two of the three patients, who went on to recover without any evidence of lasting neurologic impairment. The third patient had proximal thigh weakness after a laparotomy 1 week after operation. The progressive weakness, although not immediately reversed, was stabilized by supportive measures directed at improving spinal cord perfusion, and complete recovery occurred gradually during the ensuing month.

Renal failure. A total of 16 patients (7\%) had renal failure necessitating dialysis after resection of thoracic and thoracoabdominal aneurysms: $11 \mathrm{pa}-$ tients in group I $(12.5 \%)$ and five in group II $(5 \%)$. In nine patients, renal failure was an aspect of multisystem postoperative deterioration leading eventually to death before discharge from the hospital. Among the remaining patients, renal failure was transient in six, with only one survivor requiring long-term dialysis.

\section{Discussion}

The improvement in the results of operations for descending thoracic and thoracoabdominal aneurysms seen in our most recent cohort of patients shows that significant progress is being made, although death and paraplegia are still not sufficiently rare among patients with the most extensive aneurysms. The favorable outcome of recent operations for descending thoracic and thoracoabdominal aneurysms is likely to have been achieved principally as a combined result of specific modifications to the operative procedure and of modifications in postoperative care. There was probably some contribution from more general factors, including better anesthesia and nursing care.

The only preoperative difference between the two groups of patients that may have had a favorable influence on the better outcome in the more recent group is the inclusion of fewer patients with a significant history of smoking in this group. Because smoking is a powerful risk factor for paraplegia (but has a favorable impact on mortality), a lower prevalence of smokers may have contributed to the lower incidence of spinal cord injury in the more recent group. All the other differences between the groups did not involve risk factors that were significant predictors of mortality or paraplegia by multivariate analysis.

The most fundamental change in our approach was the introduction of SSEP monitoring for the more recent cohort of patients. Intraoperative SSEP monitoring allowed us to serially evaluate the contribution of each intersegmental artery to spinal cord function. We were initially surprised that this evaluation failed to identify any vessels that had an immediate deleterious impact on spinal cord integrity when clamped. Our interpretation of this observation is that maintenance of a high pressure in the intact descending aorta as the intercostal arteries are being sacrificed allows collateral vessels to gradually assume the function of those severed and prevents steal from the anterior spinal artery. A similar rationale has prompted Borst and $\mathrm{Laas}^{4}$ to occlude all possible intercostal arteries with vascular plugs immediately on opening the aneurysm, and he has noted a lower incidence of spinal cord injury since adopting this technique. Similarly, Acher and colleagues ${ }^{5}$ believe that their strategy of oversewing all intercostal vessels as soon as possible after the aneurysm is incised has contributed to the low incidence of spinal cord injury in their series of patients.

Because in our experience temporary clamping of intersegmental arteries never resulted in changes in the SSEPs, our technique of evaluation and stepwise elimination of inessential intersegmental vessels evolved into a policy under which no intercostal or lumbar arteries were reattached to the graft. Despite the gradual but nevertheless wholesale sacrifice of intersegmental arteries in most cases, few patients had paraplegia: none of 74 patients who had 10 or fewer pairs cut and only two of the 21 patients whose aneurysms involved sacrifice of more than 10 intersegmental arteries had permanent spinal cord injury.

These results suggest that sacrifice of 10 or fewer 
intersegmental arteries in a serial stepwise fashion is compatible with complete neurologic recovery. Ischemia to the spinal cord during operation in these cases must be mild and transient, possibly mitigated both by the presence of distal perfusion, which is used in almost all cases, and at least mild hypothermia, which decreases metabolic demands. Although the improved results in the more recent group could conceivably reflect the inclusion of a higher percentage of patients who underwent operation with HCA, which has been advocated as a means of reducing spinal cord injury by Kouchoukos and associates, ${ }^{6}$ multivariate logistic regression analysis did not support this view. Use of HCA did not emerge as a significant variable affecting development of paraplegia.

In contrast to the absence of paraplegia among patients with less extensive aneurysms, intraoperative or postoperative spinal cord injury does occasionally occur when more than 10 pairs of intersegmental arteries have been sacrificed. In some cases, the precarious circulation to the spinal cord during the first 72 hours after operation may manifest itself by transient changes in SSEPs, and our data demonstrate that the situation can often be alleviated by improving spinal cord perfusion. Draining CSF and increasing blood pressure have been successful in our hands in reversing late-onset paraplegia, and similar results have been reported by others. ${ }^{7}$ Some patients have paraplegia without warning and despite these supportive measures, however, arguing that improved methods of detecting spinal cord ischemia and of preventing spinal cord injury are still urgently required for safe operation on patients with more extensive descending thoracic and thoracoabdominal lesions.

In addition to demonstrating improved survival and lower rates of paraplegia in the recent cohort of patients with descending aortic and thoracoabdominal aneurysms, multivariate analysis involving all the patients in the study contributed to our knowledge regarding the relative risks of death and paraplegia after operation in different cases and allowed us to quantify some of these risk factors better. The presence of diabetes increases the mortality rate after operation almost fivefold, and the risk of death after operation is almost doubled if the aneurysm has ruptured. More puzzling, a history of significant smoking makes a patient one third as likely to die after operation than a comparable nonsmoker-but the smoker is more than 20 times as likely to have paraplegia.
Earlier studies have shown that smoking also predisposes the patient toward spontaneous rupture of aneurysms. ${ }^{8}$

The multivariate analysis highlights the predominant risk factor that has long been accepted empirically: the more extensive the aneurysm, the higher the risks both of paraplegia and of death after operation. The mortality rate rises progressively as the number of involved pairs of intersegmental arteries increases, and death is eight times as likely if aneurysm repair involves sacrifice of more than 10 intersegmental arteries. Paraplegia is extremely unlikely if fewer than 11 intersegmental vessels are involved, but the risk increases with each pair of vessels sacrificed and is 29 times as great if more than 10 intersegmental vessels have been severed. In this series, quantitative prediction of risk of paraplegia by number of intersegmental vessels cut was more powerful than by the widely accepted but perhaps less precise Crawford classification of thoracic and thoracoabdominal aneurysms.

The multivariate analysis thus clearly defines the group of patients with extensive thoracic and thoracoabdominal aneurysms at highest risk for development of paraplegia: those with involvement of more than 10 intersegmental arteries. In aneurysms requiring sacrifice of 10 or fewer intersegmental arteries, serial sacrifice of intersegmental arteries followed by failure to reattach any of them has not resulted in paraplegia, suggesting that the methods outlined, which emphasize gradual sacrifice and avoidance of steal phenomena, are safe and effective. It is important to note that because the highest intercostal vessel that is a branch of the aorta is the third, the group of aneurysms carrying low risk for paraplegia-those that involve 10 or fewer intersegmental arteries-includes not only all descending thoracic aneurysms ending proximal to the celiac trunk but also many thoracoabdominal aneurysms that begin in the midthoracic aorta and extend considerably more distally.

For the patients whose aneurysms involve severing more than 10 intersegmental arteries, however, it is clear that even compulsive attention to careful monitoring and to maintaining adequate perfusion pressure during and after operation cannot always prevent spinal cord injury. In this group of patients, who still had a $29 \%$ mortality and almost a $10 \%$ incidence of paraplegia in our most recent cohort, spinal cord perfusion is clearly extremely precarious. The tenuous character of postoperative spinal cord blood supply is also attested to by the $9.5 \%$ of this 
group who had late but reversible spinal cord ischemia.

In light of the failure of SSEPs to identify specific critical vessels during operation, one approach might be to add monitoring of motor-evoked potentials, which measure anterior spinal cord function, as has been advocated by Laschinger and others. ${ }^{9}$ It is possible that adding motor-evoked potentials might increase the sensitivity of our efforts to detect spinal cord ischemia during and after operation, allowing augmentation of marginal spinal cord perfusion sooner and preventing irreversible injury. Similarly, although the use of deep HCA for spinal cord protection during operation did not have a significant impact on paraplegia in this series, HCA might be helpful by markedly lowering metabolic demand during operation in patients with extensive thoracoabdominal aneurysms, reducing the metabolic debt that must be repaid during reperfusion. Our results, however, suggest that the major problem with our technique is not inadequate spinal cord protection during operation but the lack of stability of the spinal cord circulation after operation, especially when large numbers of intercostal and lumbar arteries are sacrificed. To address this problem, the routine implantation of several peridiaphragmatic intercostal vessels, as recommended by Coselli, ${ }^{10}$ may be the most promising approach.

It is important to keep in mind, however, that serial sacrifice of almost all possible intersegmental arteries without any reattachment did not result in spinal cord injury in the vast majority of our recent cases, even in patients with more extensive thoracoabdominal aneurysms. The lack of intraoperative detection of spinal cord ischemia by SSEP monitoring, together with the favorable outcomes of many patients who underwent wholesale sacrifice of almost all of their intercostal and lumbar arteries, argues against the idea that there are any specific critical intersegmental arteries that must be preserved to maintain spinal cord integrity. Instead, these excellent results even for patients with very extensive aneurysms suggest that the model of a single anterior spinal artery with multiple interchangeable inputs is more accurate than a model in which any single intersegmental artery is a prerequisite for adequate spinal cord perfusion. If no single intersegmental artery is critical, then the "artery of Adamkiewicz" is a mythical structure, and it is pointless to try to identify it either before or during operation.

\section{Conclusions}

The extremely low incidence of permanent spinal cord injury in our most recent cohort of patients suggests that serial sacrifice of intersegmental vessels, careful monitoring of spinal cord function, and a multimodal approach to maximizing spinal cord blood flow are effective in preventing paraplegia after descending thoracic and thoracoabdominal aneurysm operations. Because intraoperative SSEPs were not affected in any patient by gradual serial intersegmental artery sacrifice, the low incidence of spinal cord injury in our most recent group of patients was achieved without reimplantation of a single intercostal or lumbar artery, even when large numbers of intersegmental vessels were severed. We conclude that spinal cord blood supply is unlikely to depend on a single artery, or even on a small number of critical intersegmental vessels. We therefore question whether there is indeed an "artery of Adamkiewicz" of physiologic significance.

The low incidence of spinal cord injury, and in some cases the reversal of transient spinal cord ischemia, has also demonstrated that spinal cord perfusion can be effectively manipulated with generalized adjunctive measures without reimplantation of segmental vessels in all but a few patients with the most extensive aneurysms. In these patients at high risk, we would like to be able to combine the intraoperative advantages of our approach to serial intersegmental artery sacrifice, which allows gradual maximization of collateral flow to the anterior spinal artery and safeguards against intraoperative steal phenomena, with a technique that results in a sturdier, less tenuous spinal cord blood supply after operation, capable of withstanding minor episodes of transient systemic hypotension without precipitating obvious spinal cord ischemia. Concentrating our focus on ways to minimize intraoperative spinal cord injury and maximize spinal cord perfusion after operation in these very vulnerable patients is likely to be much more successful in eventually eliminating paraplegia than prolonging the quest for an elusive "artery of Adamkiewicz."

\section{REFERENCES}

1. Laschinger JC, Cunningham JN, Baumann FG, Cooper MM, Krieger KH, Spencer FC. Monitoring of somatosensory evoked potentials during surgical procedures on the thoracoabdominal aorta. III: intraoperative identification of vessels critical to spinal cord blood supply. J Thorac Cardiovasc Surg 1987;94:271-4.

2. Dapunt OE, Midulla PS, Sadeghi AM, Mezrow CM, Wolfe D, Gandsas A, et al. Pathogenesis of spinal cord injury during simulated aneurysm repair in a chronic animal model. Ann Thorac Surg 1994;58:689-97.

3. Galla JD, Ergin MA, Sadeghi AM, Lansman SL, Danto J, 
Griepp RB. A new technique using somatosensory evoked potential guidance during descending and thoracoabdominal aortic repairs. J Cardiac Surg 1994;9:662-72.

4. Borst HG, Laas J. Risk of replacement of descending aorta with a standardized left heart bypass technique. J Thorac Cardiovasc Surg 1994;107:126-33.

5. Acher CW, Wynn MM, Hoch JR, Popic P, Archibald J, Turnipseed, WD. Combined use of cerebrospinal fluid drainage and naloxone reduces the risk of paraplegia in thoracoabdominal aneurysm repair. J Vasc Surg 1994;19:236-46.

6. Kouchoukos NT, Daily BB, Rokkas CK, Murphy SF, Bauer $\mathrm{S}$, Abboud N. Hypothermic bypass and circulatory arrest for operations on the descending thoracic and thoracoabdominal aorta. Ann Thorac Surg 1995;60:67-77.

7. Hill AB, Kalman PG, Johnston KW, Vosu HA. Reversal of delayed-onset paraplegia after thoracic surgery with cerebrospinal fluid drainage. J Vasc Surg 1994;20:315-7.

8. Dapunt OE, Galla JD, Sadeghi AM, Lansman SL, Mezrow CK, de Asla RA, et al. The natural history of thoracic aortic aneurysms. J Thorac Cardiovasc Surg 1994;107:1323-32.

9. Laschinger JC, Owen J, Rosenbloom M, Cox JL, Kouchoukos NT. Direct noninvasive monitoring of spinal cord motor function during thoracic aortic occlusion: use of motor evoked potentials. J Vasc Surg 1988;7:161-71.

10. Coselli JS. Thoracoabdominal aortic aneurysms: experience with 372 patients. J Card Surg 1994;9:638-47.

\section{Discussion}

Dr. Joseph S. Coselli (Houston, Tex.). I congratulate you on your continued efforts to address the morbidity and mortality after operative treatment of patients with extensive thoracoabdominal aortic aneurysms, particularly with respect to the reduction in postoperative paraplegia and paraparesis. I do, however, respectfully hold a somewhat contrary view regarding the importance of the reattachment of intercostal arteries in such cases. The anatomy here, I believe, is quite important. The anterior longitudinal spinal artery provides the blood supply to the anterior two thirds of the spinal cord and is actually a vascular trunk composed of branches from the anterior radiculomedullary arteries which arise from the segmental spinal arteries, which in turn arise from the intercostal and lumbar arteries. The latter of which, in normal human anatomy, has been found to be inconsistent on multiple studies and is even more precarious in patients with diseased aortas, such as those treated surgically. The artery of Adamkiewicz has been described as the largest of these radiculomedullary arteries and most commonly arises from intercostal T8 to L2. Physiologically, however, it may actually be more than one vessel. The continuity, however, of the anterior spinal artery has been the source of differing opinions from anatomists, and consequently cannot be relied on as being a continuous vessel. The clinical and physiologic significance of the artery of Adamkiewicz is the central issue of this report. I suggest that you explore an alternative interpretation of the data regarding the importance of the reattachment of intercostal and lumbar arteries when replacing thoracoabdominal aortic aneurysms.

In an earlier experience, we reported up to $30 \%$ of postoperative paraplegia occurring as a delayed event. In our more recent experience, with an aggressive posture toward the reattachment of intercostal and lumbar arteries, delayed paraplegia has become all but nonexistent. This is consistent with the data presented here today, in which paraplegia is progressively more likely to occur as more intersegmental arteries are sacrificed. You found that the odds ratio for the risk of paraplegia in the more extensive aneurysms, those that had 11 or more intercostal arteries sacrificed, had a 29 -fold rate of paraplegia compared with cases in which 10 or fewer were sacrificed. The sacrifice of the direct spinal cord circulation places the patient in a precarious anatomic position, dependent only on collateral circulation. This is very likely a natural occurrence in many diseased aortas but should not be a situation into which the surgeon deliberately places the patient. The patient is consequently at increased risk for paraplegia, both acute and delayed, when placed in such an anatomic position. The paraplegia in the postoperative period may arise secondary to hypotension or other cardiovascular and hemodynamic fluctuations, as occurred in one patient in this series. I would concur that the spinal cord may at times be dependent upon a single segmental artery, but this may not justify or support the need to sacrifice these intercostal arteries, but rather supports their reattachment.

I have a few questions. Did you encounter any aneurysms in which ligation of all of the involved intercostal arteries before opening the aneurysm was anatomically not feasible? Were there any aneurysms that simply could not be mobilized entirely, and did any of these aneurysm mobilizations result in embolization distally? And to what do you attribute the increased mortality with an increased number of pairs of intercostal arteries ligated? Finally, how do you explain the false-negative SSEP finding in one patient you encountered in your series?

Dr. Griepp. We do not disagree that there is significant input to the spinal cord blood supply from intersegmental vessels, and I think that our data support the contention that this is the case. You can only sacrifice so many. On the other hand, the way in which you sacrifice them may have a substantial impact on how many you can get away with taking.

I would call attention to the fact that the incidence of paraplegia in this series is not zero, which is what we would all like to see: among the patients with extensive aneurysms, with 11 or more pairs of intercostal vessels sacrificed, the incidence of paraplegia was still $10 \%$. Nonetheless, $90 \%$ of these patients did not have paraplegia. I think that the challenge facing us with our particular approach is how to deal with the $10 \%$ of patients in whom we have clearly stripped away too many intersegmental feeders into the blood supply of the spinal cord, leaving the cord in a precarious position that results in paraplegia, without losing the benefit of the technique for the other $90 \%$ of patients. Attachment of intercostals in a somewhat blinded fashion, as Dr. Coselli advocates, may indeed be a reasonable alternative, and further work should be able to indicate whether that is the case.

With respect to the sacrifice of all intersegmental vessels before opening the aneurysm, there were cases in which this was not accomplished completely: a few distal vessels, particularly in the lumbar region, were not visualized until the aorta was opened. In this case, they were 
sacrificed as the first part of the procedure by being oversewn. There were, I believe, two patients in this series in whom rupture occurred during mobilization; those patients, of course, did not have any further intercostals sacrificed in a serial fashion, but intraoperative rupture is a relatively rare event.

With respect to false-negative findings in at least one case, in which the SSEPs remained normal throughout the procedure but the patient awakened paraplegic, this is a hazard of the technique. SSEPs monitor the posterior columns, and the motor nerves pass anteriorly; it is therefore certainly possible to have dissociation between these two in cord damage, and we have seen this on magnetic resonance imaging.

Dr. Robert A. Dion (Brussels, Belgium). Dr. Griepp, our group has for 5 years shared with your prestigious group a similar conception of the spinal cord monitoring. So far, we have used SSEPs in 52 procedures involving the descending aorta. Our method differs slightly from yours in that we stimulate alternately the left and the right leg and that we record the SSEPs at four levels; we believe that these maneuvers have contributed to optimizing the specificity and sensitivity of SSEP monitoring. We also use the test of time, but instead of gradually clipping the intercostal arteries from outside, we apply two clamps above and under the aortic lesion and follow the evolution of SSEPs during 15 minutes while venting the excluded aortic segment. By doing so, we probably stimulate a steal phenomenon in addition to ischemia, which may explain why, in contrast with your experience, we had to reimplant intercostal arteries in eight patients to relieve spinal cord ischemia detected by SSEPs. Four of these intercostal artery reimplantations were performed outside the territory of the Adamkiewicz artery, and we therefore also believe that this concept is a myth.

In the 44 patients displaying preoperative SSEP alteration, we did not reimplant any intercostal artery. Two of these patients had delayed paraparesis; one of them recovered. Obviously, in both patients the metabolic equilibrium of the spinal cord had been altered by a prolonged episode of relative hypotension and a sharp decrease in oxygen saturation. Ever since, even when the SSEPs do not change during operation, we would reimplant a couple of arteries if it is technically feasible, particularly in the case of an extensive aortic replacement. In our minds, this should provide some metabolic reserve for the postoperative course. In your situation, with all of the intercostal arteries clipped, how could you reverse a postoperative spinal cord ischemia detected by your continuous neural monitoring in the event that you are unable to rapidly correct a jeopardized metabolic condition?

Dr. Griepp. Thank you, Dr. Dion. I acknowledge your precedence in applying this technique. You and Dr. Cunningham did it before we did, and we took a lot of hints from you.

We considered using your particular technique of isolating segments of the aorta rather than clamping the intercostals directly, for the reasons you have indicated. It allows you to provide the more demanding stimulus of venting of the segment, actually promoting backbleeding. Dr. Dapunt in our laboratory a number of years ago showed that isolating a segment of the thoracic aorta with distal perfusion will frequently not result in spinal cord injury in the pig model, but if you actually vent that segment so that you can have blood coming out of the segmental arteries, you will then cause paraplegia. Clearly clamping and aspirating blood is a very severe test for whether those particular vessels make some contribution to spinal cord blood supply. In most of our cases, unfortunately, we did not believe that multiple clamping of the aorta was possible, owing to the presence of clot and atheroma within the vessel itself. For that reason, we decided on direct temporary occlusion of the vessels.

I certainly agree with you that the patients in whom we take many pairs of intersegmental vessels are in a precarious state. I think that one of the keys to preventing that precarious state from progressing to cord ischemia or necrosis is very careful monitoring and attention to postoperative hemodynamics. I think one of the reasons that the incidence of paraplegia is falling throughout the world in a number of centers has mainly to do with effective intraoperative techniques resulting in stable suture lines and stable hemodynamics after operation, and we will probably continue to rely on our ability to maintain a high normal blood pressure and hemodynamic stability rather than blindly doing intercostal implantation on every patient.

Dr. Hermes C. Grillo (Boston, Mass.). I have one question. Given the anatomic variability of the anterior spinal artery, its origins and major supply and collaterals, would you consider adding an anatomic factor to your clinical and physiologic study of the existence and importance of this artery? Namely, would you do spinal angiography, which can be done safely nowadays--there used to be fear of it in the old days-and then clamp the specific one that is the artery of Adamkiewicz, which I think you will find, and then measure your potentials? I do not know whether this can be done with aneurysms. We do spinal angiograms with dumbbell neurogenic tumors that lie in critical areas. This might provide the critical answer to this question. If potentials dropped, I think you would have to conclude that the artery of Adamkiewicz is not a myth. Then you would have the answer. By the way, a Polish visiting surgeon did assure us that Adamkiewicz is the correct pronunciation. We puzzled about that, too, for years.

Dr. Griepp. I do not disagree that if one does angiography or postmortem studies that one will frequently find interruptions in the anterior spinal artery, and that one can clearly find an intersegmental artery that is larger than the others, as I believe Adamkiewicz described 80 or 90 years ago. The question is whether, if you sacrifice that one vessel, other inputs into the system can provide adequate collateral flow. We certainly started out this study believing that we would find in some number of patients intercostal or lumbar vessels that, when we clamped them, would lead to clear changes in conduction in the sensory pathways. We just have not seen this so far. A negative study result does not mean that this is never going to occur, and there certainly have been cases in which a single artery has been occluded, resulting in paraplegia. Most of those cases where there were singular arteries, though, were cases in which angiography was done, and perhaps the artery was traumatized rather more than when it is just being sacrificed. There are groups that 
are looking at selective angiography. We have not pursued this because we believe that this technique is likely to be applicable only in a minority of cases. So many of these patients have large deposits of atheroma and clot within the aorta that the angiographers can delineate a few of the vessels, but they frequently have trouble delineating them all. And I think that the risk of mobilization of that atheromatous material is real. So we are looking for a technique that would be widely applicable, and for that reason we have not taken up the mantle of spinal angiography.

Dr. Chris K. Rokkas (Brookfield, Wis.). I think it is important to keep in mind that the posterior spinal columns, which are monitored by SSEPs, are not supplied by the arteria radicularis magna or artery of Adamkiewicz, therefore it may not be entirely surprising that you did not find this artery in your neurophysiologic quest. Recent experiences derived from the neurosurgical literature suggest that monitoring of magnetic, not electrical, motorevoked potentials may be a more accurate way to predict postoperative motor deficits. I wonder whether you are planning to evaluate this new technology with subsequent patients.

I also think that this is important because it is the first clinical report indicating that ischemic preconditioning of the spinal cord may indeed have a protective effect on postoperative function. We know that there are two distinct ischemic insults in spinal cord ischemic injury: spinal cord ischemic injury secondary to aortic crossclamping and spinal cord ischemic injury secondary to devascularization from interruption of the critical blood supply. It is therefore quite conceivable that gradual interruption of blood supply conditions the neuronal cells and prepares them for the more serious ischemic insult, that of the aortic crossclamping. Ischemic preconditioning in the central nervous system is known to be mediated by induction of heat shock proteins. It may be that intercostal and lumbar artery implantation is not as important if the cells at risk are preconditioned by a sublethal ischemic insult. I wonder whether you are planning to investigate this phenomenon in the laboratory further. This ischemic preconditioning phenomenon in association with the mild to moderate hypothermia that you employed in your cases may indeed account for your excellent clinical results.

Dr. Griepp. With regard to motor-evoked potentials, we have considered using them. Certainly, other investigators in this field are evaluating motor-evoked potentials as a more sensitive measure of cord function. I think at present our perception is that the SSEPs are somewhat easier to do on a routine basis, particularly in the postoperative period, so I think we will continue to use them at least for the time being and await the results of others to see whether there is indeed a better way of monitoring cord function, from both a theoretic and a practical standpoint.

With respect to ischemic preconditioning, I think that this is a very interesting idea. One would expect that if there were some ischemic preconditioning that one would see some changes, at least in the posterior columns, and we have occasionally seen slight increases in latency that have then returned to baseline within a few minutes after clamping of some intercostal vessels. It is thus possible that there is a cellular explanation for what we have seen, in addition to an anatomic one. Our primary interpretation has been that by occluding these vessels gradually and obviating altogether the possibility of steal, one allows other collateral pathways to gradually dilate during the period of serial sacrifice, explaining our result, but the cellular level is another interesting avenue to pursue.

\section{Availability of Journal back issues}

As a service to our subscribers, copies of back issues of The Journal of Thoracic and Cardiovascular Surgery for the preceding 5 years are maintained and are available for purchase from Mosby at a cost of $\$ 13.50$ per issue until inventory is depleted. The following quantity discounts are available: $25 \%$ off on quantities of 12 to 23 , and one third off on quantities of 24 or more. Please write to Mosby-Year Book, Inc., Subscription Services, 11830 Westline Industrial Drive, St. Louis MO 63146-3318, or call 800-453-4351 or 314-453-4351 for information on availability of particular issues. If unavailable from the publisher, photocopies of complete issues may be purchased from UMI, 300 N. Zeeb Rd., Ann Arbor, MI 48106, 313-761-4700. 